\title{
Infinitely Many Quasi-Coincidence Point Solutions of Multivariate Polynomial Problems
}

\author{
Yi-Chou Chen \\ Department of General Education, National Army Academy, Taoyuan 320, Taiwan \\ Correspondence should be addressed to Yi-Chou Chen; cycu.chou@gmail.com \\ Received 9 November 2013; Accepted 5 December 2013 \\ Academic Editor: Wei-Shih Du
}

Copyright ( 2013 Yi-Chou Chen. This is an open access article distributed under the Creative Commons Attribution License, which permits unrestricted use, distribution, and reproduction in any medium, provided the original work is properly cited.

Let $F: \mathbb{R}^{n} \times \mathbb{R} \rightarrow \mathbb{R}$ be a real-valued polynomial function of the form $F(\bar{x}, y)=a_{s}(\bar{x}) y^{s}+a_{s-1}(\bar{x}) y^{s-1}+\cdots+a_{0}(\bar{x})$ where the degree $s$ of $y$ in $F(\bar{x}, y)$ is greater than 1. For arbitrary polynomial function $f(\bar{x}) \in \mathbb{R}[\bar{x}], \bar{x} \in \mathbb{R}^{n}$, we will find a polynomial solution $y(\bar{x}) \in \mathbb{R}[\bar{x}]$ to satisfy the following equation $(\star): F(\bar{x}, y(\bar{x}))=a f(\bar{x})$ where $a \in \mathbb{R}$ is a constant depending on the solution $y(\bar{x})$, namely a quasi-coincidence (point) solution of $(\star)$, and $a$ is called a quasi-coincidence value of $(\star)$. In this paper, we prove that (i) the number of all solutions in $(\star)$ does not exceed $\operatorname{deg}_{y} F(\bar{x}, y)\left(\left(2^{\operatorname{deg} f(\bar{x})}+s+3\right) \cdot 2^{\operatorname{deg} f(\bar{x})}+1\right)$ provided those solutions are of finitely many exist, (ii) if all solutions are of infinitely many exist, then any solution is represented as the form $y(\bar{x})=-a_{s-1}(\bar{x}) / s a_{s}(\bar{x})+\lambda p(\bar{x})$ where $\lambda$ is arbitrary and $p(\bar{x})=\left(f(\bar{x}) / a_{s}(\bar{x})\right)^{1 / s}$ is also a factor of $f(\bar{x})$, provided the equation $(\star)$ has infinitely many quasi-coincidence (point) solutions.

\section{Introduction}

In 1987, Lenstra [1] researched a polynomial function $F(x, y) \in \mathbb{Q}(\alpha)[x, y](\alpha$ is an algebraic number) and attempted to search the factorization of $F(x, y)$. Continuing his job, many scientists tried to find the roots of the polynomial equations (cf. [2-6]). Later, many authors also studied fixed point theory and fixed coincidence theory (cf. [7-11]). Recently, Lai and Chen ([12-15]) research the quasi-fixed (point) polynomial problem; they assumed $F: \mathbb{R}^{n} \times \mathbb{R} \rightarrow \mathbb{R}$ a polynomial function and solved $y(x) \in \mathbb{R}[x]$ to satisfy the polynomial equation as the form

$$
F(x, y(x))=a p^{m}(x), \quad x \in \mathbb{R},
$$

where $a \in \mathbb{R}, p(x)$ is an irreducible polynomial in $\mathbb{R}[x]$, and the polynomial function $F(x, y)$ is written by

$$
F(x, y)=\sum_{i=0}^{s} a_{i}(x) y^{i} \quad \text { with } s \geq 1,
$$

where $s=\operatorname{deg}_{y} F$ denotes the degree of $y$ in $F(x, y)$.

Definition 1 (Lai and Chen [12]). A polynomial function $y=y(x)$ satisfying (1) is called a quasi-fixed solution corresponding to some real number $a$. This number $a$ is called a quasi-fixed value corresponding to the polynomial solutions $y=y(x)$.

Moreover, Chen and Lai [16] extended (1) to a more general coincidence (point) problem in which the $f(x) \in \mathbb{R}[x]$ is replaced by the irreducible polynomial power $p^{m}(x) \in \mathbb{R}[x]$, where $f(x)$ is an arbitrary polynomial. Then we restate (1) as the following equation:

$$
F(x, y)=a f(x) .
$$

It is a new development coincidence point-like problem. We call the polynomial solution $y=y(x)$ for (3) a quasicoincidence (point) solution. Precisely, we give the following definition like Definition 1.

Definition 2 (Chen and Lai [16]). A polynomial function $y=y(x)$ satisfying (3) is called a quasi-coincidence (point) solution corresponding to some real number $a$. This number $a$ is called a quasi-coincidence value corresponding to the polynomial solutions $y=y(x)$. 
Furthermore, we consider a multivariate polynomial function $F: \mathbb{R}^{n} \times \mathbb{R} \rightarrow \mathbb{R}$ and extend (3) as a more general coincidence (point) problem in which the $\bar{x}=\left(x_{1}, x_{2}, \ldots, x_{n}\right)$ is replaced by $x$ throughout this paper, where $f(\bar{x})$ is a nonzero arbitrary polynomial in $\mathbb{R}[\bar{x}]$. Then we restate (3) as the following equation:

$$
F(\bar{x}, y)=a f(\bar{x}) .
$$

Thus, we can give some definitions like Definition 2 as follows.

Definition 3. A polynomial function $y=y(\bar{x})$ satisfying (4) is called a quasi-coincidence (point) solution corresponding to some real number $a$. This number $a$ is called a quasicoincidence value corresponding to the polynomial solutions $y=y(\bar{x})$.

The number of all solutions in (4) may be infinitely many, finitely many, or not solvable. In this paper, we solve all solutions of (4) if the number is infinitely many. Moreover, we provide an upper bound for the number of all solutions if the number is finitely many.

In Section 2, we derive some properties of quasicoincidence solutions. If (4) has infinitely many quasicoincidence solutions, the form of $F(\bar{x}, y)$ will be described in Section 3. In the last section, we solve all solutions if (4) has infinitely many solutions.

\section{Preliminaries}

For convenience, we denote the polynomial function by

$$
\begin{aligned}
F(\bar{x}, y) & =a_{s}(\bar{x}) y^{s}+a_{s-1}(\bar{x}) y^{s-1}+\cdots+a_{1}(\bar{x}) y+a_{0}(\bar{x}) \\
& =\sum_{i=0}^{s} a_{i}(\bar{x}) y^{i}
\end{aligned}
$$

throughout this paper and since there may exist many solutions corresponding to the same number $a$, we use the similar notations like (Definition 2, [11]) to represent them.

Notation 1. (1) $\mathrm{Qcs}_{F}$, the set of all solutions satisfying equation (4), the solution in $\mathrm{Qcs}_{F}$ is also called a quasicoincidence solution in (4) (like Definition 2).

(2) $\mathrm{Qcv}_{F}$, the set of all solutions $a$ satisfying equation (4), the solution in $\mathrm{Qcv}_{F}$ is also called a quasi-coincidence value in (4) (like Definition 2).

(3) $\operatorname{Qcs}_{F}(a)$, the set of all quasi-coincidence solutions $y(\bar{x})$ corresponding to a quasi-coincidence value $a$.

(4) For each $a \in \mathbb{R}$, we denote $\left|\mathrm{Qcs}_{F}(a)\right|$ as the cardinal number of $\operatorname{Qcs}_{F}(a)$.

Evidently, by Notation 1, we have the following lemma.

Lemma 4. (i) $Q c s_{F}=\bigcup_{a \in \operatorname{Qcv}_{F}} Q c s_{F}(a)$;

(ii) $Q c s_{F}(a) \cap Q c s_{F}(b)=\emptyset$ for any $a \neq b$ in $Q c v_{F}$;

(iii) $\left|Q c s_{F}(a)\right| \leq \operatorname{deg}_{y} F(\bar{x}, y)$ for any $a \in \mathbb{R}$;

(iv) $\left|Q c s_{F}\right| \leq\left|Q c v_{F}\right|\left|Q c s_{F}(a)\right|$ for any $a \in Q c v_{F}$.
Proof. (i) $\bigcup_{a \in \mathrm{Qcv}_{F}} \mathrm{Qcs}_{F}(a) \subseteq \mathrm{Qcs}_{F}$ is obvious.

Conversely, for any $y(\bar{x}) \in \mathrm{Qcs}_{F}$, by Notation 1(1), we have

$$
F(\bar{x}, y(\bar{x}))=a f(\bar{x})
$$

for some $a \in \operatorname{Qcv}_{F}$. This means $y(\bar{x}) \in \operatorname{Qcs}_{F}(a)$ and we obtain

$$
\operatorname{Qcs}_{F} \subseteq \bigcup_{a \in \operatorname{Qcv}_{F}} \operatorname{Qcs}_{F}(a) .
$$

(ii) Let $a \neq b$ in $\mathrm{Qcv}_{F}$; if there exists $y(\bar{x}) \in \mathbb{R}[\bar{x}]$ such that

$$
y(\bar{x}) \in \operatorname{Qcs}_{F}(a) \bigcap \operatorname{Qcs}_{F}(b),
$$

by Notation 1(3), we have

$$
\text { af }(\bar{x})=F(\bar{x}, y(\bar{x}))=b f(\bar{x}) .
$$

This leads a contradiction to $a \neq b$ and we have $\operatorname{Qcs}_{F}(a) \bigcap \operatorname{Qcs}_{F}(b)=\emptyset$.

(iii) For each $a \in \mathbb{R}$, the number of all solutions $y=$ $y(\bar{x})$ to the polynomial equation $F(\bar{x}, y)-a f(\bar{x})=0$ is at most $\operatorname{deg}_{y}(F(\bar{x}, y)-a f(\bar{x}))=\operatorname{deg}_{y} F(\bar{x}, y)$; then the result is obtained.

(iv) By (i), we have

$$
\mathrm{Qcs}_{F}=\bigcup_{a \in \mathrm{Qcv}_{F}} \operatorname{Qcs}_{F}(a) .
$$

It follows that

$$
\begin{aligned}
\left|\mathrm{Qcs}_{F}\right| & =\left|\bigcup_{a \in \operatorname{Qcv}_{F}} \operatorname{Qcs}_{F}(a)\right| \\
& \leq\left|\operatorname{Qcv}_{F}\right|\left|\operatorname{Qcs}_{F}(a)\right|, \quad \text { for any } a \in \operatorname{Qcv}_{F} .
\end{aligned}
$$

In the following lemma, we explain some interesting properties of the relations of quasi-coincidence point solutions. Throughout this paper, we consider (4) for polynomial function (5) and nonzero arbitrary polynomial $f(\bar{x})$ in $\mathbb{R}[\bar{x}]$.

Lemma 5. Let the cardinal number $\left|Q c v_{F}\right| \geq 2$ and $a \neq b$ in $Q c v_{F}$. Then for any $y_{1}(\bar{x}) \in Q c s_{F}(a)$ and $y_{2}(\bar{x}) \in Q c s_{F}(b)$, one has

$$
y_{1}(\bar{x})-y_{2}(\bar{x})=d p(\bar{x}) \quad \text { for some } d \in \mathbb{R},
$$

and this $p(\bar{x})$ is a factor of $f(\bar{x})$, that is, $p(\bar{x}) \mid f(\bar{x})$.

Proof. Since $y_{1}(\bar{x}), y_{2}(\bar{x})$ correspond to $a, b$, respectively, we have

$$
\begin{aligned}
& F\left(\bar{x}, y_{1}(\bar{x})\right)=a f(\bar{x}), \\
& F\left(\bar{x}, y_{2}(\bar{x})\right)=b f(\bar{x}) .
\end{aligned}
$$


Subtracting (14) from (13) and using binomial formula, it yields that

$$
\begin{aligned}
(a-b) f(\bar{x})= & F\left(\bar{x}, y_{1}(\bar{x})\right)-F\left(\bar{x}, y_{2}(\bar{x})\right) \\
= & a_{s}(\bar{x})\left[y_{1}^{s}(\bar{x})-y_{2}^{s}(\bar{x})\right] \\
& +a_{s-1}(\bar{x})\left[y_{1}^{s-1}(\bar{x})-y_{2}^{s-1}(\bar{x})\right] \\
& +\cdots+a_{1}(\bar{x})\left[y_{1}(\bar{x})-y_{2}(\bar{x})\right] \\
= & {\left[y_{1}(\bar{x})-y_{2}(\bar{x})\right] } \\
& \times\left[a_{s}(\bar{x}) G_{s}\left(y_{1}(\bar{x}), y_{2}(\bar{x})\right)\right. \\
& \quad+a_{s-1}(\bar{x}) G_{s-1}\left(y_{1}(\bar{x}), y_{2}(\bar{x})\right) \\
& \left.\quad+\cdots+a_{1}(\bar{x})\right] \\
= & {\left[y_{1}(\bar{x})-y_{2}(\bar{x})\right] Q\left(x, y_{1}(\bar{x}), y_{2}(\bar{x})\right), }
\end{aligned}
$$

where

$$
\begin{aligned}
G_{j}\left(y_{1}(\bar{x}), y_{2}(\bar{x})\right)= & y_{1}^{j-1}(\bar{x}) \\
& +y_{1}^{j-2}(\bar{x}) y_{2}(\bar{x})+\cdots+y_{2}^{j-1}(\bar{x}),
\end{aligned}
$$

for $j=s, s-1, \ldots, 2,1$. Evidently, the factor $y_{1}(\bar{x})-y_{2}(\bar{x})$ is divisible to the term $(a-b) f(\bar{x})$ and since $a \neq b$, we obtain

$$
y_{1}(\bar{x})-y_{2}(\bar{x})=d p(\bar{x})
$$

for some real number $d \in \mathbb{R}$ and factor $p(\bar{x})$ of $f(\bar{x})$.

In Lemma 5, the difference of any two distinct quasicoincidence solutions corresponding to distinct values is a factor of $f(\bar{x})$. Thus we may define a class of those factors in the following.

Notation 2. (i) Denote $\Phi(p(\bar{x}))=\{\alpha p(\bar{x}): \alpha \in \mathbb{R}\}$.

(ii) Let $y(\bar{x})$ be an arbitrary polynomial in $\mathbb{R}[\bar{x}]$, and we denote $y(\bar{x})+\Phi(p(\bar{x}))=\{y(\bar{x})+\alpha p(\bar{x}): \alpha \in \mathbb{R}\}$.

(iii) $\operatorname{deg} f(\bar{x})=\sum_{i=1}^{n} \operatorname{deg}_{x_{i}} f(\bar{x})$.

If $y_{1}(x), y_{2}(x) \in \mathrm{Qcs}_{F}$ correspond to distinct quasicoincidence values, by Lemma 5 and Notation 2, we have

$$
y_{1}(x)-y_{2}(x) \in \bigcup_{p(\bar{x}) \mid f(\bar{x})} \Phi(p(\bar{x})) .
$$

Since the number of all factors $p(\bar{x})$ to $f(\bar{x})$ is at most $2^{\operatorname{deg} f(\bar{x})}$, by the definitions of "the pigeonhole principle" in [17], we have the following results.

Lemma 6. Suppose that

$$
\left|Q c v_{F}\right| \geq k \cdot 2^{\operatorname{deg} f(\bar{x})}+2 .
$$

Then there exists $y(\bar{x}) \in Q c s_{F}$ and $p(\bar{x})$ is a factor of $f(\bar{x})$ such that

$$
\left|(y(\bar{x})+\Phi(p(\bar{x}))) \cap Q c s_{F}\right| \geq k .
$$

Proof. Since $\left|\mathrm{Qcv}_{F}\right| \geq k 2^{\operatorname{deg} f(\bar{x})}+2$, by (18), there exists $y_{1}(\bar{x})$, $y_{2}(\bar{x}), \ldots, y_{\left\{k 2^{\operatorname{deg} f(\bar{x})}+2\right\}}(\bar{x}) \in \operatorname{Qcs}_{F}$ such that

$$
y_{j}(\bar{x})-y_{1}(\bar{x}) \in \Phi\left(p_{j}(\bar{x})\right)
$$

for some factor $p_{j}(\bar{x})$ of $f(\bar{x})$, for $j=2,3, \ldots, k 2^{\operatorname{deg} f(\bar{x})}+2$. Moreover, we have that the number of all factors to $f(\bar{x})$ is at most $2^{\operatorname{deg} f(\bar{x})}$. By "the pigeonhole principle," there exists a subset $\left\{y_{j_{i}}(\bar{x})\right\}_{i=1}^{k} \subseteq\left\{y_{j}(\bar{x})\right\}_{j=2}^{k 2^{\operatorname{deg} f(\bar{x})}+2}$ such that

$$
y_{j_{i}}(\bar{x})-y_{1}(\bar{x}) \in \Phi(p(\bar{x})),
$$

for $i=1,2, \ldots, k$ and some factor $p(\bar{x})$ of $F(\bar{x})$ (this $p(\bar{x})$ is independent of the choice of $i$ ). Thus

$$
\begin{gathered}
\left\{y_{j_{i}}(\bar{x})\right\}_{i=1}^{k} \subseteq\left(y_{1}(\bar{x})+\Phi(p(\bar{x}))\right) \cap \operatorname{Qcs}_{F}, \\
\left|\left(y_{1}(\bar{x})+\Phi(p(\bar{x}))\right) \cap \mathrm{Qcs}_{F}\right| \geq k .
\end{gathered}
$$

For convenience, we explain the relations of $\mathrm{Qcs}_{F}$ and $\Phi(p(\bar{x}))$ in the following lemma.

Lemma 7. Let $y(\bar{x}) \in \operatorname{Qcs}_{F}(a)$ for some $a \in \mathbb{R}$. Then

$Q c s_{F}=\operatorname{Qcs}_{F}(a) \bigcup\left(\bigcup_{p(\bar{x}) \mid f(\bar{x})}\left\{(y(\bar{x})+\Phi(p(\bar{x}))) \cap Q c s_{F}\right\}\right)$

for some factor $p(\bar{x})$ of $f(\bar{x})$.

Proof. For any $y_{1}(\bar{x}) \in \operatorname{Qcs}_{F} \backslash \operatorname{Qcs} F(a)$, then $y_{1}(\bar{x}) \in \operatorname{Qcs}(b)$ for some $b \in \mathrm{Qcv}_{F}$. By Lemma 5, we have

$$
y_{1}(\bar{x})-y(\bar{x}) \in \Phi(p(\bar{x}))
$$

for some factor $p(\bar{x})$ of $f(\bar{x})$. Then

$$
y_{1}(\bar{x}) \in \bigcup_{p(\bar{x}) \mid f(\bar{x})}\{y(\bar{x})+\Phi(p(\bar{x}))\}
$$

and it follows that

$$
\operatorname{Qcs}_{F} \subseteq \operatorname{Qcs}_{F}(a) \bigcup\left(\bigcup_{p(\bar{x}) \mid f(\bar{x})}\{y(\bar{x})+\Phi(p(\bar{x}))\}\right) .
$$

Moreover, by Lemma 4(i), $\operatorname{Qcs}_{F}(a) \subseteq \operatorname{Qcs}_{F}$; then we obtain

$$
\begin{aligned}
& \operatorname{Qcs}_{F} \\
& \quad=\operatorname{Qcs}_{F}(a) \bigcup\left(\bigcup_{p(\bar{x}) \mid f(\bar{x})}\left\{(y(\bar{x})+\Phi(p(\bar{x}))) \cap \operatorname{Qcs}_{F}\right\}\right) .
\end{aligned}
$$

In order to let the number of all elements in the intersection of sets $y_{1}(\bar{x})+\Phi\left(p_{1}(\bar{x})\right)$ and $y_{2}(\bar{x})+\Phi\left(p_{2}(\bar{x})\right)$ be large enough, we find a lower bound for $\left|\mathrm{Qcv}_{F}\right|$ in the following theorem. 
Theorem 8. Suppose that the cardinal number

$$
\left|Q c v_{F}\right| \geq\left(2^{\operatorname{deg} f(\bar{x})}+s+3\right)\left(2^{\operatorname{deg} f(\bar{x})}\right)+2
$$

Then for any $y_{1}(\bar{x}) \neq y_{2}(\bar{x}) \in Q c s_{F}$, there exist two factors $p_{1}(\bar{x})$ and $p_{2}(\bar{x})$ of $f(\bar{x})$ such that

$$
\begin{aligned}
& \mid\left(y_{1}(\bar{x})+\Phi\left(p_{1}(\bar{x})\right)\right) \cap\left(y_{2}(\bar{x})+\Phi\left(p_{2}(\bar{x})\right)\right) \cap Q c s_{F} \\
& \quad-\left\{y_{1}(\bar{x}), y_{2}(\bar{x})\right\} \mid \geq 2 .
\end{aligned}
$$

Proof. Let $y_{1}(\bar{x}) \in \mathrm{Qcs}_{F}$ and by assumption

$$
\left|\mathrm{Qcv}_{F}\right| \geq\left(2^{\operatorname{deg} f(\bar{x})}+s+3\right)\left(2^{\operatorname{deg} f(\bar{x})}\right)+2,
$$

and by Lemma 6 , there exists a factor $p_{1}(\bar{x})$ of $f(\bar{x})$ such that

$$
\left|\left(y_{1}(\bar{x})+\Phi\left(p_{1}(\bar{x})\right)\right) \cap \mathrm{Qcs}_{F}\right| \geq 2^{\operatorname{deg} f(\bar{x})}+s+3 .
$$

This implies that

$$
\left|\left(y_{1}(\bar{x})+\Phi\left(p_{1}(\bar{x})\right)\right) \cap \mathrm{Qcs}_{F}-\left\{y_{1}(\bar{x})\right\}\right| \geq 2^{\operatorname{deg} f(\bar{x})}+s+2 .
$$

Moreover, for any $y_{2}(\bar{x}) \in \mathrm{Qcs}_{F}$, we have

$$
\begin{aligned}
& \left(y_{1}(\bar{x})+\Phi\left(p_{1}(\bar{x})\right)\right) \\
& \quad \cap \operatorname{Qcs}_{F}-\left\{y_{1}(\bar{x})\right\} \subseteq\left(y_{1}(\bar{x})+\Phi\left(p_{1}(\bar{x})\right)\right) \cap \operatorname{Qcs}_{F} \\
& \quad \subseteq \operatorname{Qcs}_{F}
\end{aligned}
$$

(by Lemma 7)

$$
=\operatorname{Qcs}_{F}(b) \bigcup\left(\bigcup_{p(\bar{x}) \mid f(\bar{x})}\left(y_{2}(\bar{x})+\Phi(p(\bar{x}))\right) \cap \operatorname{Qcs}_{F}\right),
$$

for some constant $b \in \mathbb{R}$ and it follows that

$$
\begin{aligned}
& 2^{\operatorname{deg}} f(\bar{x})+s+2 \\
& \quad=\left|\left(y_{1}(\bar{x})+\Phi\left(p_{1}(\bar{x})\right)\right) \cap \mathrm{Qcs}_{F}-\left\{y_{1}(\bar{x})\right\}\right| \\
& \quad \leq\left|\left(y_{1}(\bar{x})+\Phi\left(p_{1}(\bar{x})\right)\right) \cap \mathrm{Qcs}_{F}\right| \\
& \quad \leq\left|\mathrm{Qcs}{ }_{F}(b) \bigcup\left(\bigcup_{p(\bar{x}) \mid f(\bar{x})}\left(y_{2}(\bar{x})+\Phi(p(\bar{x}))\right) \cap \mathrm{Qcs}_{F}\right)\right| \\
& \quad \leq\left|\operatorname{Qcs}_{F}(b)\right|+\sum_{p(\bar{x}) \mid f(\bar{x})}\left|\left(y_{2}(\bar{x})+\Phi(p(\bar{x}))\right) \cap \operatorname{Qcs}_{F}\right|
\end{aligned}
$$

(by Lemma 4 (iii))

$$
\leq s+\sum_{p(\bar{x}) \mid f(\bar{x})}\left|y_{2}(\bar{x})+\Phi(p(\bar{x})) \cap \mathrm{Qcs}_{F}\right| .
$$

Canceling both sides of the above inequality by " $s$ ", it follows that

$$
2^{\operatorname{deg} f(\bar{x})}+2 \leq \sum_{p(\bar{x}) \mid f(\bar{x})}\left|y_{2}(\bar{x})+\Phi(p(\bar{x})) \cap \mathrm{Qcs}_{F}\right|,
$$

this implies that

$$
2^{\operatorname{deg} f(\bar{x})}+1 \leq \sum_{p(\bar{x}) \mid f(\bar{x})}\left|y_{2}(\bar{x})+\Phi(p(\bar{x})) \cap \operatorname{Qcs}_{F}-\left\{y_{2}(\bar{x})\right\}\right| ;
$$

By the pigeonhole' principle and since the number of all factors $p(\bar{x})$ to $f(\bar{x})$ is at most $2^{\operatorname{deg} f(\bar{x})}$, we have

$$
\begin{gathered}
\left|\left(y_{2}(\bar{x})+\Phi\left(p_{2}(\bar{x})\right)\right) \cap \mathrm{Qcs}_{F}-\left\{y_{2}(\bar{x})\right\}\right| \geq 2, \\
\mid\left(y_{1}(\bar{x})+\Phi\left(p_{1}(\bar{x})\right)-\left\{y_{1}(\bar{x})\right\}\right) \\
\quad \cap\left(y_{2}(\bar{x})+\Phi\left(p_{2}(\bar{x})\right)-\left\{y_{2}(\bar{x})\right\}\right) \cap \mathrm{Qcs}_{F} \mid \geq 2
\end{gathered}
$$

for some factor $p_{2}(\bar{x})$ of $f(\bar{x})$. Thus we obtain

$$
\begin{aligned}
& \mid\left(y_{1}(\bar{x})+\Phi\left(p_{1}(\bar{x})\right)\right) \cap\left(y_{2}(\bar{x})+\Phi\left(p_{2}(\bar{x})\right)\right) \cap \mathrm{Qcs}_{F} \\
& \quad-\left\{y_{1}(\bar{x}), y_{2}(\bar{x})\right\} \mid \geq 2 .
\end{aligned}
$$

Up to now, we have not shown that the factor $p(\bar{x})$ uniquely existed eventually. In the following theorem, we would show the uniqueness property for the factor $p(\bar{x})$ of $f(\bar{x})$ if the number of all quasi-coincidence values is large enough.

Theorem 9. Assume that the cardinal number

$$
\left|Q c v_{F}\right| \geq\left(2^{\operatorname{deg} f(\bar{x})}+s+3\right)\left(2^{\operatorname{deg} f(\bar{x})}\right)+2 .
$$

Then for any $y_{1}(\bar{x}), y_{2}(\bar{x}) \in Q c s_{F}$, one has

$$
y_{1}(\bar{x})-y_{2}(\bar{x})=\lambda p(\bar{x}),
$$

where $\lambda \in \mathbb{R}$ and $p(\bar{x})$ is a factor of $f(\bar{x})$ (this $p(\bar{x})$ is independent of the choice of $y_{1}(\bar{x})$ and $\left.y_{2}(\bar{x})\right)$.

Proof. Let $y_{1}(\bar{x}) \neq y_{2}(\bar{x}) \in \mathrm{Qcs}_{F}$; by Theorem 8 , we have

$$
\begin{aligned}
& \mid\left(y_{1}(\bar{x})+\Phi\left(p_{1}(\bar{x})\right)\right) \cap\left(y_{2}(\bar{x})+\Phi\left(p_{2}(\bar{x})\right)\right) \cap \mathrm{Qcs}_{F} \\
& \quad-\left\{y_{1}(\bar{x}), y_{2}(\bar{x})\right\} \mid \geq 2
\end{aligned}
$$

for some factors $p_{1}(\bar{x})$ and $p_{2}(\bar{x})$ of $f(\bar{x})$. There exists $g_{1}(\bar{x}) \neq g_{2}(\bar{x}) \in \mathrm{Qcs}_{F}$, such that

$$
\begin{aligned}
g_{1}(\bar{x}), g_{2}(\bar{x}) & \in\left(y_{1}(\bar{x})+\Phi\left(p_{1}(\bar{x})\right)\right) \\
& \cap\left(y_{2}(\bar{x})+\Phi\left(p_{2}(\bar{x})\right)\right) \\
& \cap \operatorname{Qcs}_{F}-\left\{y_{1}(\bar{x}), y_{2}(\bar{x})\right\} .
\end{aligned}
$$

This implies that

$$
\begin{aligned}
& g_{1}(\bar{x}) \in y_{1}(\bar{x})+\Phi\left(p_{1}(\bar{x})\right), \\
& g_{2}(\bar{x}) \in y_{1}(\bar{x})+\Phi\left(p_{1}(\bar{x})\right), \\
& g_{1}(\bar{x}) \in y_{2}(\bar{x})+\Phi\left(p_{2}(\bar{x})\right), \\
& g_{2}(\bar{x}) \in y_{2}(\bar{x})+\Phi\left(p_{2}(\bar{x})\right) .
\end{aligned}
$$


By Notation 2(ii), it yields that

$$
\begin{aligned}
& g_{1}(\bar{x})-y_{1}(\bar{x})=\lambda_{1} p_{1}(\bar{x}), \\
& g_{2}(\bar{x})-y_{1}(\bar{x})=\lambda_{2} p_{1}(\bar{x}), \\
& g_{1}(\bar{x})-y_{2}(\bar{x})=\lambda_{3} p_{2}(\bar{x}), \\
& g_{2}(\bar{x})-y_{2}(\bar{x})=\lambda_{4} p_{2}(\bar{x})
\end{aligned}
$$

for some constants $\lambda_{1}, \lambda_{2}, \lambda_{3}$, and $\lambda_{4} \in \mathbb{R}$ and consequently

$$
\begin{aligned}
g_{2}(\bar{x})-g_{1}(\bar{x}) & =\left(g_{2}(\bar{x})-y_{1}(\bar{x})\right)-\left(g_{1}(\bar{x})-y_{1}(\bar{x})\right) \\
& =\left(\lambda_{2}-\lambda_{1}\right) p_{1}(\bar{x}), \\
g_{2}(\bar{x})-g_{1}(\bar{x}) & =\left(g_{2}(\bar{x})-y_{2}(\bar{x})\right)-\left(g_{1}(\bar{x})-y_{2}(\bar{x})\right) \\
& =\left(\lambda_{4}-\lambda_{3}\right) p_{2}(\bar{x}) .
\end{aligned}
$$

This implies that

$$
\left(\lambda_{2}-\lambda_{1}\right) p_{1}(\bar{x})=\left(\lambda_{4}-\lambda_{3}\right) p_{2}(\bar{x})
$$

and $p_{1}(\bar{x})=p_{2}(\bar{x})$. Therefore,

$$
\begin{aligned}
y_{1}(\bar{x})-y_{2}(\bar{x}) & =\left(g_{1}(\bar{x})-y_{2}(\bar{x})\right)-\left(g_{1}(\bar{x})-y_{1}(\bar{x})\right) \\
& =\left(\lambda_{3}-\lambda_{1}\right) p_{1}(\bar{x}),
\end{aligned}
$$

and this means that the factor $p(\bar{x})$ of $f(\bar{x})$ is uniquely determined independent of the choice of $y_{1}(\bar{x})$ and $y_{2}(\bar{x})$.

Corollary 10. Assume that the cardinal number

$$
\left|Q c s_{F}\right| \geq \operatorname{deg}_{y} F(\bar{x}, y)\left(\left(2^{\operatorname{deg} f(\bar{x})}+s+3\right)\left(2^{\operatorname{deg} f(\bar{x})}\right)+2\right) .
$$

Then for any $y(\bar{x}) \in Q c s_{F}$, there exists $h(\bar{x}), p(\bar{x}) \in \mathbb{R}[\bar{x}]$ such that

$$
y(\bar{x})=h(\bar{x})+\lambda p(\bar{x})
$$

for some $\lambda \in \mathbb{R}(h(\bar{x}), p(\bar{x})$ are independent of the choice of $y(\bar{x}))$.

Proof. By Lemma 4(iv), we have

$$
\left|\mathrm{Q} c s_{F}\right| \leq\left|\mathrm{Qcv}_{F}\right|\left|\mathrm{Qcs}_{F}(a)\right|, \quad \text { for any } a \in \mathrm{Qcv}_{F}
$$

(by Lemma 4 (iii))

$$
\leq \operatorname{deg}_{y} F(\bar{x}, y)\left|\mathrm{Qcv}_{F}\right| \text {. }
$$

By assumption, it follows that

$$
\begin{aligned}
& \operatorname{deg}_{y} F(\bar{x}, y)\left(\left(2^{\operatorname{deg} f(\bar{x})}+s+3\right)\left(2^{\operatorname{deg} f(\bar{x})}\right)+2\right) \\
& \leq \operatorname{deg}_{y} F(\bar{x}, y)\left|\mathrm{Qcv}_{F}\right| .
\end{aligned}
$$

Dividing both sides of the above equation by $\operatorname{deg}_{y} F(\bar{x}, y)$, we get

$$
\left|\mathrm{Qcv}_{F}\right| \geq\left(\left(2^{\operatorname{deg} f(\bar{x})}+s+3\right)\left(2^{\operatorname{deg} f(\bar{x})}\right)+2\right)
$$

If $h(\bar{x}) \in \operatorname{Qcs}_{F}$, for any $y(\bar{x}) \in \mathrm{Qcs}_{F}$, by Theorem 9, we have

$$
y(\bar{x})=h(\bar{x})+d p(\bar{x})
$$

for some factor $p(\bar{x})$ of $f(\bar{x})$.

\section{The Type of $F(\bar{x}, y)$ If the Number of All Quasi-Coincidence Solutions Is Infinitely Many}

In this section, we consider (4) for polynomial function $F(\bar{x}, y)$ in (5); that is, let

$$
F(\bar{x}, y)=\sum_{i=0}^{s} a_{i}(\bar{x}) y^{i} \quad \text { with } s \geq 2 .
$$

$f(\bar{x}) \in \mathbb{R}[\bar{x}]$ and we assume that $F(\bar{x}, y)$ has at least $s+1$ distinct quasi-coincidence solutions satisfying some conditions, that is, $y_{1}(\bar{x}), y_{2}(\bar{x}), y_{3}(\bar{x}), \ldots, y_{s+1}(\bar{x}), \ldots$ in the following theorem. According to the above assumptions, we could derive the following result.

Theorem 11. Suppose that the cardinal number $\left|Q c s_{F}\right| \geq s+1$ and for each $y(\bar{x}) \in Q c s_{F}$ can be represented as the form

$$
y(\bar{x})=y_{1}(\bar{x})+\lambda_{i} p(\bar{x}), \quad \lambda_{i} \in \mathbb{R},
$$

for some $y_{1}(\bar{x}), p(\bar{x}) \in \mathbb{R}[\bar{x}]$ and $\lambda_{i} \in \mathbb{R}$, for $i=1,2, \ldots, s+$ $1, \ldots$. Then $p^{s}(\bar{x}) \mid f(\bar{x})$ and the polynomial $F(\bar{x}, y)$ can be represented as

$$
F(\bar{x}, y)=\sum_{i=0}^{s} c_{i} \frac{f(\bar{x})}{p^{i}(\bar{x})}\left(y-y_{1}(\bar{x})\right)^{i}
$$

for constants $c_{i} \in \mathbb{R}, i=0,1, \ldots, s$.

Proof. Let $y_{i}(\bar{x})$ be distinct quasi-coincidence solutions of $F(\bar{x}, y)$ corresponding to quasi-coincidence values $a_{i}, 1 \leq i \leq$ $s+1$, such that

$$
F\left(\bar{x}, y_{i}(\bar{x})\right)=a_{i} f(\bar{x})
$$

Choose $i=1, F\left(\bar{x}, y_{1}(\bar{x})\right)=a_{1} f(\bar{x})$. When $y-y_{1}(\bar{x})$ divides the function $F(\bar{x}, y)$, we get

$$
F(\bar{x}, y)=\left(y-y_{1}(\bar{x})\right) F_{1}(\bar{x}, y)+a_{1} f(\bar{x}),
$$

where $F_{1}(\bar{x}, y)$ is the quotient and $a_{1} f(\bar{x})$ is the remainder. From the above identity, take $y=y_{2}(\bar{x})$, and it becomes

$$
\begin{aligned}
F\left(\bar{x}, y_{2}(\bar{x})\right) & =\left(y_{2}(\bar{x})-y_{1}(\bar{x})\right) F_{1}\left(\bar{x}, y_{2}(\bar{x})\right)+a_{1} f(\bar{x}) \\
& =a_{2} f(\bar{x}) .
\end{aligned}
$$


Then

$$
\left(y_{2}(\bar{x})-y_{1}(\bar{x})\right) F_{1}\left(\bar{x}, y_{2}(\bar{x})\right)=\left(a_{2}-a_{1}\right) f(\bar{x}) .
$$

By (56), $y_{2}(\bar{x})-y_{1}(\bar{x})=\lambda_{2} p(\bar{x})$, it yields that

$$
\begin{aligned}
F_{1}\left(\bar{x}, y_{2}(\bar{x})\right) & =\left(\frac{\left(a_{2}-a_{1}\right)}{\lambda_{2}}\right) \frac{f(\bar{x})}{p(\bar{x})} \\
& =d_{2} \frac{f(\bar{x})}{p(\bar{x})} \in \mathbb{R}[\bar{x}] \quad \text { for } d_{2}=\frac{\left(a_{2}-a_{1}\right)}{\lambda_{2}} .
\end{aligned}
$$

Hence

$$
F_{1}(\bar{x}, y)=\left(y-y_{2}(\bar{x})\right) F_{2}(\bar{x}, y)+d_{2} \frac{f(\bar{x})}{p(\bar{x})} .
$$

Continuing this process from $i=2$ to $s-1$, we obtain

$$
F_{i}(\bar{x}, y)=\left(y-y_{i+1}(\bar{x})\right) F_{i+1}(x, y)+d_{i+1} \frac{f(\bar{x})}{p^{i}(\bar{x})},
$$

for some $d_{i+1} \in \mathbb{R}, i=1,2, \ldots, s-1$. Finally, we could get

$$
F_{s-1}(\bar{x}, y)=\left(y-y_{s}(\bar{x})\right) F_{s}(\bar{x})+d_{s} \frac{f(\bar{x})}{p^{s-1}(\bar{x})} .
$$

$F_{s}(\bar{x})$ does not contain the variable $y$ since $\operatorname{deg}_{y} F=s$. By the assumption (58), $F\left(\bar{x}, y_{s+1}(\bar{x})\right)=a_{s+1} f(\bar{x})$. It follows that

$$
F_{s}(\bar{x})=\lambda \frac{f(\bar{x})}{p^{s}(\bar{x})} \in \mathbb{R}[\bar{x}], \quad \text { for some constant } \lambda \in \mathbb{R} \text {. }
$$

Consequently,

$$
\begin{aligned}
& F(\bar{x}, y)=\left(y-y_{1}(\bar{x})\right) F_{1}(\bar{x}, y)+a_{1} f(\bar{x}) \\
& =\left(y-y_{1}(\bar{x})\right)\left(\left(y-y_{2}(\bar{x})\right) F_{2}(\bar{x}, y)+d_{2} \frac{f(\bar{x})}{p(\bar{x})}\right) \\
& +a_{1} f(\bar{x})=\cdots=\left(y-y_{1}(\bar{x})\right) \\
& \times\left(\left(y-y_{2}(\bar{x})\right)\right. \\
& \times\left(\cdots\left(\left(y-y_{s}(\bar{x})\right) F_{s}(\bar{x})+d_{s} \frac{f(\bar{x})}{p^{s-1}(\bar{x})}\right) \cdots\right) \\
& \left.+d_{2} \frac{f(\bar{x})}{p(\bar{x})}\right)+a_{1} f(\bar{x}) \\
& =\left(y-y_{1}(\bar{x})\right) \\
& \times\left(\left(y-y_{2}(\bar{x})\right)\right. \\
& \times\left(\cdots \left(\left(y-y_{s}(\bar{x})\right) \lambda \frac{f(\bar{x})}{p^{s}(\bar{x})}\right.\right. \\
& \left.\left.\left.+d_{s} \frac{f(\bar{x})}{p^{s-1}(\bar{x})}\right) \cdots\right)+d_{2} \frac{f(\bar{x})}{p(\bar{x})}\right) \\
& +a_{1} f(\bar{x}) .
\end{aligned}
$$

By (56), we have $y_{i}(\bar{x})=y_{1}(\bar{x})+\lambda_{i} p(\bar{x}), i=2,3, \ldots, s+1$. Then $F(\bar{x}, y)$ can be expanded to a power series in the expression

$$
\begin{aligned}
F(\bar{x}, y)=( & \left.y-y_{1}(\bar{x})\right) \\
& \times\left(\left(y-y_{1}(\bar{x})-\lambda_{2} p(\bar{x})\right)\right. \\
& \times\left(\cdots \left(\left(y-y_{1}(\bar{x})-\lambda_{s} p(\bar{x})\right) \lambda \frac{f(\bar{x})}{p^{s}(\bar{x})}\right.\right. \\
& \left.\left.\left.+d_{s} \frac{f(\bar{x})}{p^{s-1}(\bar{x})}\right) \cdots\right)+d_{2} \frac{f(\bar{x})}{p(\bar{x})}\right) \\
& +a_{1} f(\bar{x})=\sum_{i=0}^{s} c_{i} \frac{f(\bar{x})}{p^{i}(\bar{x})}\left(y-y_{1}(\bar{x})\right)^{i},
\end{aligned}
$$

for some real numbers $c_{j}, j=0,1, \ldots, s$. Moreover, the leading coefficient of $F(\bar{x}, y), c_{s}\left(f(\bar{x}) / p^{s}(\bar{x})\right)$ is contained to $\mathbb{R}[\bar{x}]$, and it follows $p^{s}(\bar{x}) \mid f(\bar{x})$.

In the above theorem, if there exist at least $s+1$ quasicoincidence solutions with some relations, then $F(\bar{x}, y)$ has a fixed type. In the following theorem, if $F(\bar{x}, y)$ has a fixed type expressed as in Theorem 11, then the cardinal number $\left|\mathrm{Q} \mathrm{cs}_{F}\right|=\infty$.

Theorem 12. The following three conditions are equivalent:

(i) $F(\bar{x}, y)=\sum_{i=0}^{s} c_{i}\left(f(\bar{x}) / p^{i}(\bar{x})\right)\left(y-y_{1}(\bar{x})\right)^{i}$ for some $y_{1}(\bar{x}) \in \mathbb{R}[\bar{x}]$, some factor $p(\bar{x})$ of $f(\bar{x})$ and $c_{i} \in \mathbb{R}$, $i=0,1, \ldots, s$;

(ii) $\left|Q c s_{F}\right|=\infty$;

(iii) $\left|Q c v_{F}\right| \geq\left(2^{\operatorname{deg} f(\bar{x})}+s+3\right) \cdot 2^{\operatorname{deg} f(\bar{x})}+2$.

(In fact, if $\left|Q c s_{F}\right|=\infty$, then $\left|Q c s_{F}\right|=$ the cardinal number of $\mathbb{R})$.

Proof. (i) $\Rightarrow$ (ii) Suppose that (i) holds. Let $y=y_{1}(\bar{x})+\lambda p(\bar{x})$ for any constant $\lambda \in \mathbb{R}$; we have

$$
\begin{aligned}
F\left(\bar{x}, y_{1}(\bar{x})+\lambda p(\bar{x})\right) & =\sum_{i=0}^{s} c_{i} \frac{f(\bar{x})}{p^{i}(\bar{x})}(\lambda p(\bar{x}))^{i} \\
& =\left(\sum_{i=0}^{s} c_{i} \lambda^{i}\right) f(\bar{x}) \\
& =c p^{m}(\bar{x}) \quad \text { for } c=\sum_{i=0}^{s} c_{i} \lambda^{i} \in \mathbb{R} .
\end{aligned}
$$

This means that $y_{1}(\bar{x})+\lambda p(\bar{x}) \in \mathrm{Qcs}_{F}$ for all $\lambda \in \mathbb{R}$ and we obtain

$$
\infty=\left|\left\{y_{1}(\bar{x})+\lambda p(\bar{x})\right\}_{\lambda \in \mathbb{R}}\right| \leq \mid \mathrm{Qcs} F
$$

It follows that the cardinal number $\left|\mathrm{Q} \mathrm{cs}_{F}\right|=\infty$. 
(ii) $\Rightarrow$ (iii) can be obtained obvious from Lemma 4(iv). have

(iii) $\Rightarrow$ (i) For any $y(\bar{x}), y_{1}(\bar{x}) \in \mathrm{Qcs}_{F}$, by Theorem 9 , we

$$
y(\bar{x})-y_{1}(\bar{x})=d p(\bar{x})
$$

for some fixed factor $p(\bar{x})$ of $f(\bar{x})$ and by Theorem 11 , we obtain

$$
F(\bar{x}, y)=\sum_{i=0}^{s} c_{i} \frac{f(\bar{x})}{p^{i}(\bar{x})}\left(y-y_{1}(\bar{x})\right)^{i}
$$

for some $y_{1}(\bar{x})$ and $c_{i} \in \mathbb{R}, i=0,1, \ldots, s$.

Corollary 13. If the number of all quasi-fixed solutions is finitely many, the number of all quasi-fixed values does not exceed an integer $\ell$. Actually,

$$
\ell=\left(2^{\operatorname{deg} f(\bar{x})}+s+3\right) \cdot 2^{\operatorname{deg} f(\bar{x})}+1
$$

Proof. By the contrapositive of Theorem 12(ii) $\Rightarrow$ (iii), we have "if $|\mathrm{Qcs} F|<\infty$, then $\left|\mathrm{Qcv}_{F}\right|<\left(2^{\operatorname{deg} f(\bar{x})}+s+3\right) \cdot 2^{\operatorname{deg} f(\bar{x})}+$ 2." Hence the number of all quasi-fixed values is at most $\ell$; that is, $\ell \leq\left(2^{\operatorname{deg} f(\bar{x})}+s+3\right) \cdot 2^{\operatorname{deg} f(\bar{x})}+1$.

Corollary 14. If the number of all quasi-fixed solutions is finitely many, the number of all quasi-fixed solutions does not exceed

$$
\operatorname{deg}_{y} F(\bar{x}, y)\left(\left(2^{\operatorname{deg} f(\bar{x})}+s+3\right) \cdot 2^{\operatorname{deg} f(\bar{x})}+1\right) .
$$

Proof. By Lemma 4(iv), we have for any $a \in \mathrm{Qcv}_{F}$

$$
\left|\mathrm{Qcs}_{F}\right| \leq\left|\mathrm{Qcs}_{F}(a)\right|\left|\mathrm{Qcv}_{F}\right|
$$

(by Lemma 4 (iii) and Corollary 13)

$$
\leq \operatorname{deg}_{y} F(\bar{x}, y)\left(\left(2^{\operatorname{deg} f(\bar{x})}+s+3\right) \cdot 2^{\operatorname{deg} f(\bar{x})}+1\right) .
$$

\section{Main Theorems and Some Corollaries}

If the $F(\bar{x}, y)$ can be represented as the form (57), then any quasi-coincidence solution can be formed in this section.

Lemma 15. Let $F(\bar{x}, y)$ be represented as in $(57)$. Then $h(\bar{x}) \epsilon$ $\mathbb{R}[\bar{x}]$ is a quasi-coincidence solution of $F(\bar{x}, y)$ if and only if

$$
h(\bar{x})=y(\bar{x})+d p(\bar{x})
$$

for some $d \in \mathbb{R}$ and some factor $p(\bar{x})$ of $f(\bar{x})$.

Proof. Since

$$
F(\bar{x}, y)=\sum_{i=0}^{s} c_{i} \frac{f(\bar{x})}{p^{i}(\bar{x})}(y-y(\bar{x}))^{i}
$$

we let $y=y(\bar{x})$, and then

$$
\begin{aligned}
F(\bar{x}, y(\bar{x})) & =\sum_{i=0}^{s} c_{i} \frac{f(\bar{x})}{p^{i}(\bar{x})}(y(\bar{x})-y(\bar{x}))^{i} \\
& =c_{0} f(\bar{x}) ;
\end{aligned}
$$

this means $y(\bar{x}) \in \mathrm{Qcs}_{F}$.

By Theorem 12,

$$
F(\bar{x}, y)=\sum_{i=0}^{s} c_{i} \frac{f(\bar{x})}{p^{i}(\bar{x})}(y-y(\bar{x}))^{i}, \quad \text { then }\left|\mathrm{Qcs}_{F}\right|=\infty .
$$

Assume that $h(\bar{x})$ is a quasi-coincidence solution of $F(\bar{x}, y)$ and by Corollary 10 , we obtain that for any quasi-coincidence solution $h(\bar{x})$, we have

$$
h(\bar{x})=y(\bar{x})+d p(\bar{x}), \quad \text { for some } d \in \mathbb{R} .
$$

Conversely, suppose $h(\bar{x})=y(\bar{x})+d p(\bar{x})$, for some factor $p(\bar{x})$ of $f(\bar{x})$ and $d \in \mathbb{R}$. Substituting this $h(\bar{x})$ as $y$ in (57), we have

$$
\begin{aligned}
F(\bar{x}, h(\bar{x})) & =F(\bar{x}, y(\bar{x})+d p(\bar{x})) \\
& =\sum_{i=0}^{s} c_{i} \frac{f(\bar{x})}{p^{i}(\bar{x})}(d p(\bar{x}))^{i} \\
& =\left(\sum_{i=0}^{s} c_{i} d^{i}\right) f(\bar{x}) .
\end{aligned}
$$

Therefore $h(\bar{x}) \in \mathrm{Qcs}_{F}$.

Note that not any polynomial function $F(\bar{x}, y)$ can be written as (57). Actually, almost all $F(\bar{x}, y)$ are expressed as the form of the next theorem. In this situation, any solution can be written as the next form $(*)$ in this theorem under some condition.

Theorem 16. Let $F(\bar{x}, y)$ be a polynomial function with

$$
F(\bar{x}, y)=a_{s}(\bar{x}) y^{s}+a_{s-1}(\bar{x}) y^{s-1}+\cdots+a_{0}(\bar{x}),
$$

and $f(\bar{x})$ a polynomial. If the cardinal number $\left|Q c s_{F}\right|$ is infinitely many, then each quasi-coincidence solution of (4) must be of the form

$$
-\frac{a_{s-1}(\bar{x})}{s a_{s}(\bar{x})}+\lambda p(\bar{x})
$$

for arbitrary $\lambda \in \mathbb{R}$, where $p(\bar{x})=\left(f(\bar{x}) / a_{s}(\bar{x})\right)^{1 / s}$ is a factor of $f(\bar{x})$.

Proof. Assume $\left|\mathrm{Q} \mathrm{cs}_{F}\right|=\infty$. By Theorem 12, we have

$$
\begin{aligned}
F(\bar{x}, y) & =a_{s}(\bar{x}) y^{s}+a_{s-1}(\bar{x}) y^{s-1}+\cdots+a_{0}(\bar{x}) \\
& =\sum_{i=0}^{s} c_{i} \frac{f(\bar{x})}{p^{i}(\bar{x})}(y-y(\bar{x}))^{i},
\end{aligned}
$$


for some $c_{i} \in \mathbb{R}, i=0, \ldots, s$, and $y(\bar{x}) \in \operatorname{Qcs}_{F}$. Comparing the coefficients of $y^{s}$ and $y^{s-1}$ in both sides of the above equation, we get

$$
\begin{gathered}
a_{s}(\bar{x})=c_{s} \frac{f(\bar{x})}{p^{s}(\bar{x})}, \\
a_{s-1}(\bar{x})=-s a_{s}(\bar{x}) y(\bar{x})+c_{s-1} \frac{f(\bar{x})}{p^{s-1}(\bar{x})} .
\end{gathered}
$$

Consequently, by (84), we get

$$
\begin{gathered}
p^{s}(\bar{x})=c_{s} \frac{f(\bar{x})}{a_{s}(\bar{x})}, \\
y(\bar{x})=\frac{c_{s-1}}{s c_{s}} p(\bar{x})-\frac{a_{s-1}(\bar{x})}{s a_{s}(\bar{x})} \in \mathbb{R}[\bar{x}] .
\end{gathered}
$$

By Lemma 15, for any $d \in \mathbb{R}$, we have that any quasicoincidence solution is represented by

$$
\begin{aligned}
y(\bar{x})+d p(\bar{x}) & =\frac{c_{s-1}}{s c_{s}} p(\bar{x})-\frac{a_{s-1}(\bar{x})}{s a_{s}(\bar{x})}+d p(\bar{x}) \\
& =-\frac{a_{s-1}(\bar{x})}{s a_{s}(\bar{x})}+\left(d-\frac{c_{s-1}}{s c_{s}}\right) p(\bar{x}) \\
& =-\frac{a_{s-1}(\bar{x})}{s a_{s}(\bar{x})}+\lambda p(\bar{x})
\end{aligned}
$$

where $p(\bar{x})=\left(c_{s}\right)^{1 / s}\left(f(\bar{x}) / a_{s}(\bar{x})\right)^{1 / s}$ (note that since $d$ is arbitrary, then $\lambda$ is arbitrary).

This completes the proof.

Corollary 17. Let $F(\bar{x}, y)$ be a polynomial function with

$$
F(\bar{x}, y)=a_{s}(\bar{x}) y^{s}+a_{s-1}(\bar{x}) y^{s-1}+\cdots+a_{0}(\bar{x}) .
$$

If the cardinal number $\left|Q c s_{F}\right|$ of equation

$$
F(\bar{x}, y)=a
$$

is infinitely many, then the leading coefficient $a_{s}(\bar{x})$ must be a real number and each quasi-coincidence point solution must be of the form

$$
\lambda-\frac{a_{s-1}(\bar{x})}{s a_{s}(\bar{x})}
$$

for arbitrary $\lambda \in \mathbb{R}$.

Corollary 18. Let $F: \mathbb{R} \times \mathbb{R} \rightarrow \mathbb{R}$ be a polynomial function with

$$
F(x, y)=a_{s}(x) y^{s}+a_{s-1}(x) y^{s-1}+\cdots+a_{0}(x) .
$$

If the cardinal number $\left|Q c s_{F}\right|$ of equation

$$
F(x, y)=a x
$$

is infinitely many, then the leading coefficient $a_{s}(x)$ must be a real number and each quasi-coincidence point solution must be of the form

$$
\lambda_{1}+\lambda_{2} \frac{a_{s-1}(x)}{x}
$$

for arbitrary $\lambda_{1}$ and some $\lambda_{2} \in \mathbb{R}$.
Proof. Assume that there exist infinitely many solutions; by Theorem 16, any solution of (4) has the form

$$
-\frac{a_{s-1}(x)}{s a_{s}(x)}+\lambda p(x)
$$

for arbitrary $\lambda \in \mathbb{R}$ and $p(x)=c\left(x / a_{s}(x)\right)^{1 / s} \in \mathbb{R}[x]$, and then $a_{s}(x)$ is a factor of $x$. This means that $a_{s}(\bar{x}) \in \mathbb{R}$ or $a_{s}(\bar{x})=k x$ for some constant $k \in \mathbb{R}$, if $a_{s}(x) \in \mathbb{R}$; this implies $p(x)=c\left(x / a_{s}(x)\right)^{1 / s} \notin \mathbb{R}[x]$, and this leads a contradiction. So we have $a_{s}(x)=k x$, for some $k \in \mathbb{R}$; then $p(x)=c / k^{1 / s} \in \mathbb{R}$ and any solution (91) is represented as

$$
-\frac{a_{s-1}(x)}{s a_{s}(x)}+\lambda p(x)=\lambda_{1}+\lambda_{2} \frac{a_{s-1}(x)}{x}
$$

for arbitrary $\lambda_{1}=\lambda c / k^{1 / s}$ and some $\lambda_{2}=-1 / s k \in \mathbb{R}$.

Finally, we provide one example to explain Theorem 16.

Example 19. Let $\bar{x}=\left(x_{1}, x_{2}\right) \in \mathbb{R}^{2}, f(\bar{x})=\left(x_{1}+x_{2}\right)^{2}$, and

$$
\begin{aligned}
F(\bar{x}, y)= & a_{2}(\bar{x}) y^{2}+a_{1}(\bar{x}) y+a_{0}(\bar{x}) \\
= & y^{2}-\left(2 x_{1} x_{2}-x_{1}-x_{2}\right) y \\
& +\left(x_{1}^{2} x_{2}^{2}-x_{1}^{2} x_{2}-x_{1} x_{2}^{2}+x_{1}^{2}+2 x_{1} x_{2}+x_{2}^{2}\right) .
\end{aligned}
$$

Can we solve all quasi-fixed solutions of $F(\bar{x}, y)=a f(\bar{x})$ ? This polynomial function has exactly $5(\geq s+3$, since $s=2)$ quasi-fixed solutions as follows:

$$
\begin{array}{r}
F\left(x_{1}, x_{2}, x_{1} x_{2}-x_{1}-x_{2}\right)=1\left(x_{1}+x_{2}\right)^{2}, \\
F\left(x_{1}, x_{2}, x_{1} x_{2}\right)=1\left(x_{1}+x_{2}\right)^{2}, \\
F\left(x_{1}, x_{2}, x_{1} x_{2}+x_{1}+x_{2}\right)=3\left(x_{1}+x_{2}\right)^{2}, \\
F\left(x_{1}, x_{2}, x_{1} x_{2}+2 x_{1}+2 x_{2}\right)=3\left(x_{1}+x_{2}\right)^{2}, \\
F\left(x_{1}, x_{2}, x_{1} x_{2}+\frac{x_{1}}{2}+\frac{x_{2}}{2}\right)=\frac{3}{4}\left(x_{1}+x_{2}\right)^{2} .
\end{array}
$$

In fact, by Theorem 16, we can find any quasi-coincidence solution written as

$$
\begin{aligned}
-\frac{a_{1}(\bar{x})}{s a_{2}(\bar{x})}+\lambda p(\bar{x}) & =\frac{2 x_{1} x_{2}-x_{1}-x_{2}}{2}+\lambda p(\bar{x}) \\
& =x_{1} x_{2}+\left(\lambda-\frac{1}{2}\right)\left(x_{1}-x_{2}\right) \\
& =x_{1} x_{2}+\mu\left(x_{1}-x_{2}\right),
\end{aligned}
$$

where $\mu=\lambda-1 / 2 \in \mathbb{R}$ is arbitrary and $p(\bar{x})=$ $\left(f(x) / a_{s}(x)\right)^{1 / s}=x_{1}+x_{2}$. This shows the quasi-coincidence (point) solutions have cardinal $\left|\mathrm{Q} s_{F}\right|=\infty$.

In practice, we have no idea to check the number of this equation is infinitely many or finitely many. But we provide an easy method to solve all solutions if the number of all 
solutions is infinitely many in this paper. Thus, we can solve those solutions directly and check whether those solutions are the solutions of $F(\bar{x}, y)=a f(\bar{x})$ and give an example in the following.

Example 20. Let $\bar{x}=\left(x_{1}, x_{2}\right) \in \mathbb{R}^{2}, f(\bar{x})=x_{1}^{2}\left(x_{2}+x_{3}\right)^{2}$, and

$$
\begin{aligned}
F(\bar{x}, y)= & a_{2}(\bar{x}) y^{2}+a_{1}(\bar{x}) y+a_{0}(\bar{x}) \\
= & y^{2}-\left(2 x_{1} x_{2}-x_{1}\left(x_{2}+x_{3}\right)\right) y \\
& +\left(x_{1}^{2} x_{2}^{2}+x_{1}^{2} x_{2} x_{3}+x_{1}^{2} x_{3}^{2}\right) .
\end{aligned}
$$

We will solve all quasi-fixed solutions of $F(\bar{x}, y)=a f(\bar{x})$ if the number of all solutions is infinitely many.

By Theorem 16, we can find that any quasi-coincidence solution can be written as

$$
\begin{aligned}
-\frac{a_{1}(\bar{x})}{s a_{2}(\bar{x})}+\lambda p(\bar{x}) & =\frac{2 x_{1} x_{2}-x_{1}\left(x_{2}+x_{3}\right)}{2}+\lambda p(\bar{x}) \\
& =x_{1} x_{2}+\mu x_{1}\left(x_{2}+x_{3}\right),
\end{aligned}
$$

where $\mu=\lambda-1 / 2 \in \mathbb{R}$ is arbitrary and $p(\bar{x})=$ $\left(f(x) / a_{s}(x)\right)^{1 / s}=x_{1}\left(x_{2}+x_{3}\right)$. We let $y(\bar{x})=x_{1} x_{2}+\mu x_{1}\left(x_{2}+\right.$ $x_{3}$ ) and calculate $F(x, y(\bar{x}))$ and obtain

$$
F(x, y(\bar{x}))=\left(\mu^{2}+\mu+1\right) x_{1}^{2}\left(x_{2}+x_{3}\right)^{2} .
$$

This means that the quasi-coincidence (point) solutions have cardinal $\left|Q s_{F}\right|=\infty$.

We would like to provide one open problem as follows.

Further Development. Let $\mathbb{Q}$ be a quotient field. Consider a quotient-valued polynomial function

$$
F: \mathbb{Q}^{n} \times \mathbb{Q} \longrightarrow \mathbb{Q}
$$

Can we find all quasi-coincidence solutions $y=y(\bar{x}) \in \mathbb{Q}[\bar{x}]$ to satisfy

$$
F(\bar{x}, y)=a f(\bar{x})
$$

for some polynomials $f(\bar{x}) \in \mathbb{Q}[\bar{x}]$ by a co-NP hardness algorithm?

\section{References}

[1] A. K. Lenstra, "Factoring multivariate polynomials over algebraic number fields," SIAM Journal on Computing, vol. 16, no. 3 , pp. 591-598, 1987.

[2] E. P. Tsigaridas and I. Z. Emiris, "Univariate polynomial real root isolation: continued fractions revisited," in AlgorithmsESA 2006, vol. 4168 of Lecture Notes in Computer Science, pp. 817-828, Springer, Berlin, Germany, 2006.

[3] J. von zur Gathen and J. Gerhard, Modern Computer Algebra, Cambridge University Press, Cambridge, UK, 2nd edition, 2003.

[4] M. G. Marinari, H. M. Möller, and T. Mora, "On multiplicities in polynomial system solving," Transactions of the American Mathematical Society, vol. 348, no. 8, pp. 3283-3321, 1996.
[5] P. Gopalan, V. Guruswami, and R. J. Lipton, "Algorithms for modular counting of roots of multivariate polynomials," Algorithmica, vol. 50, no. 4, pp. 479-496, 2008.

[6] V. Y. Pan, "Univariate polynomials: nearly optimal algorithms for numerical factorization and root-finding," Journal of Symbolic Computation, vol. 33, no. 5, pp. 701-733, 2002.

[7] C.-M. Chen, T.-H. Chang, and Y.-P. Liao, "Coincidence theorems, generalized variational inequality theorems, and minimax inequality theorems for the $\phi$-mapping on $G$-convex spaces," Fixed Point Theory and Applications, vol. 2007, Article ID 078696, 13 pages, 2007.

[8] C.-M. Chen, T.-H. Chang, and C.-W. Chung, "Coincidence theorems on nonconvex sets and its applications," The Taiwanese Journal of Mathematics, vol. 13, no. 2, pp. 501-513, 2009.

[9] W.-S. Du, "On coincidence point and fixed point theorems for nonlinear multivalued maps," Topology and Its Applications, vol. 159, no. 1, pp. 49-56, 2012.

[10] W.-S. Du, "On approximate coincidence point properties and their applications to fixed point theory," Journal of Applied Mathematics, vol. 2012, Article ID 302830, 17 pages, 2012.

[11] W.-S. Du and S.-X. Zheng, "New nonlinear conditions and inequalities for the existence of coincidence points and fixed points," Journal of Applied Mathematics, vol. 2012, Article ID 196759, 12 pages, 2012.

[12] H.-C. Lai and Y.-C. Chen, "A quasi-fixed polynomial problem for a polynomial function," Journal of Nonlinear and Convex Analysis, vol. 11, no. 1, pp. 101-114, 2010.

[13] H.-C. Lai and Y.-C. Chen, "Quasi-fixed polynomial for vectorvalued polynomial functions on $\mathbb{R}^{n} \times \mathbb{R}$," Fixed Point Theory, vol. 12, no. 2, pp. 391-400, 2011 (Romanian).

[14] Y. C. Chen and H. C. Lai, "Quasi-fixed point solutions of realvalued polynomial equation," in Proceedings of the 9th International Conference on Fixed Point Theory and Its Applications, pp. 16-22, Changhua, Taiwan, 2009.

[15] Y.-C. Chen and H.-C. Lai, "A non-NP-complete algorithm for a quasi-fixed polynomial problem," Abstract and Applied Analysis, vol. 2013, Article ID 893045, 10 pages, 2013.

[16] Y. C. Chen and H. C. Lai, "New quasi-coincidence point polynomial problems," Journal of Applied Mathematics, vol. 2013, Article ID 959464, 8 pages, 2013.

[17] R. P. Grimaldi, Discrete and Combinatorial Mathematics, Pearson, Secaucus, NJ, USA, 5th edition, 2003. 


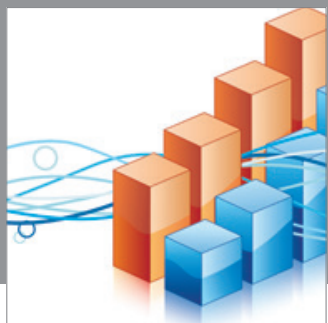

Advances in

Operations Research

mansans

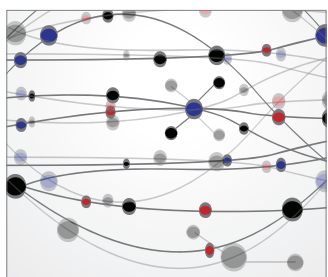

The Scientific World Journal
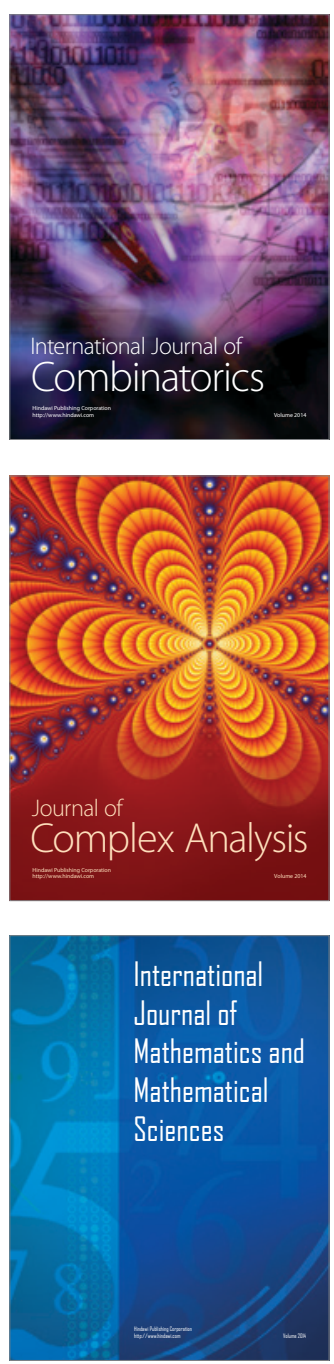
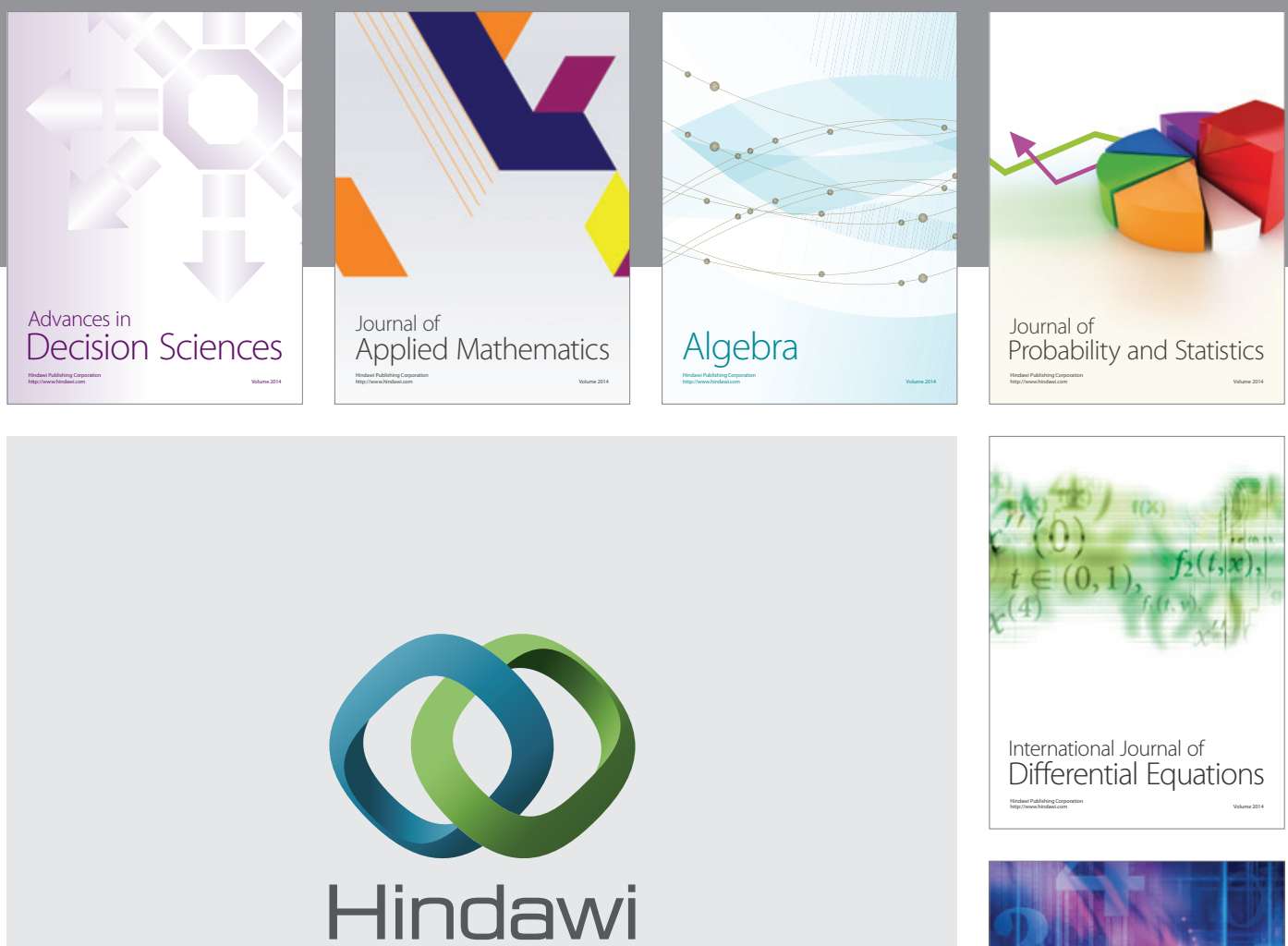

Submit your manuscripts at http://www.hindawi.com
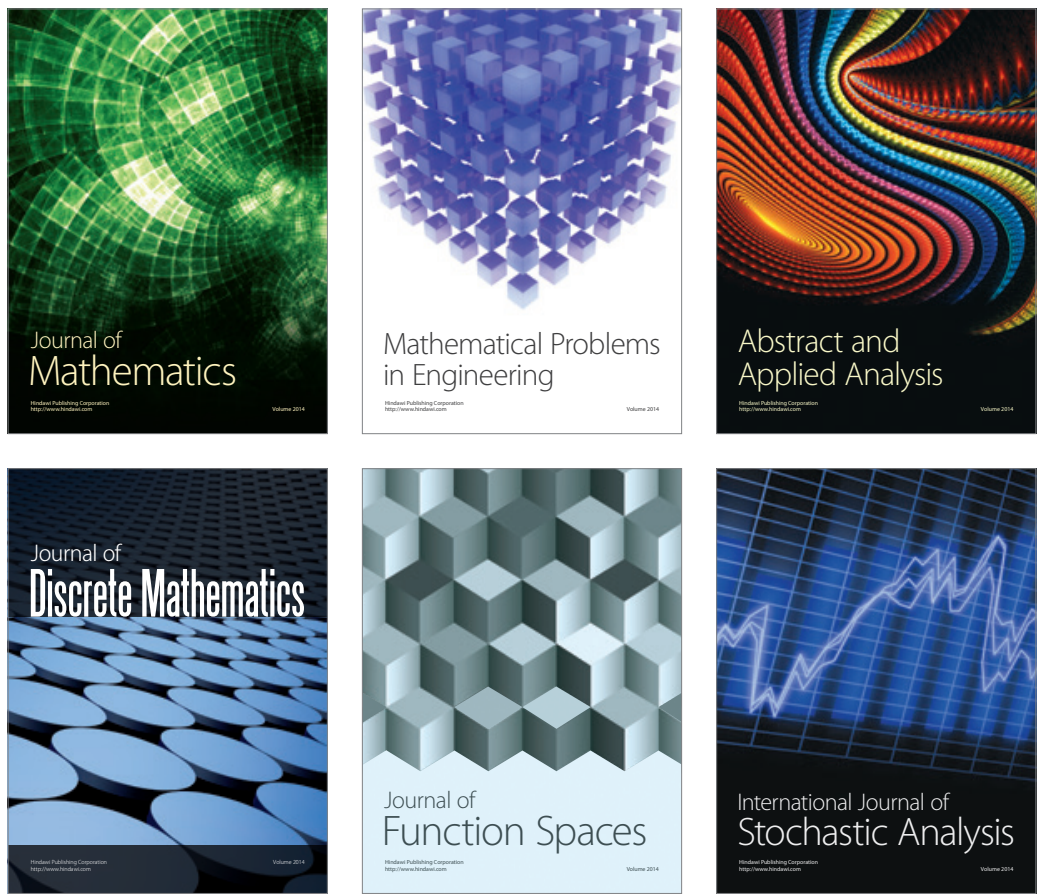

Journal of

Function Spaces

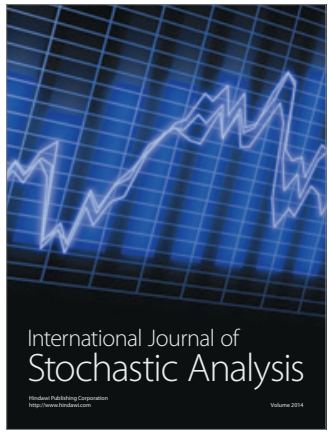

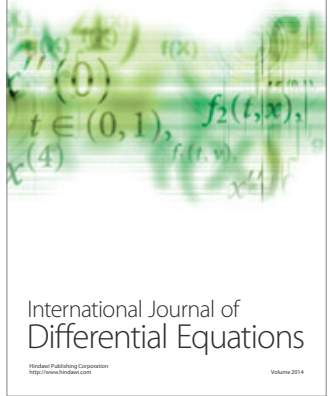
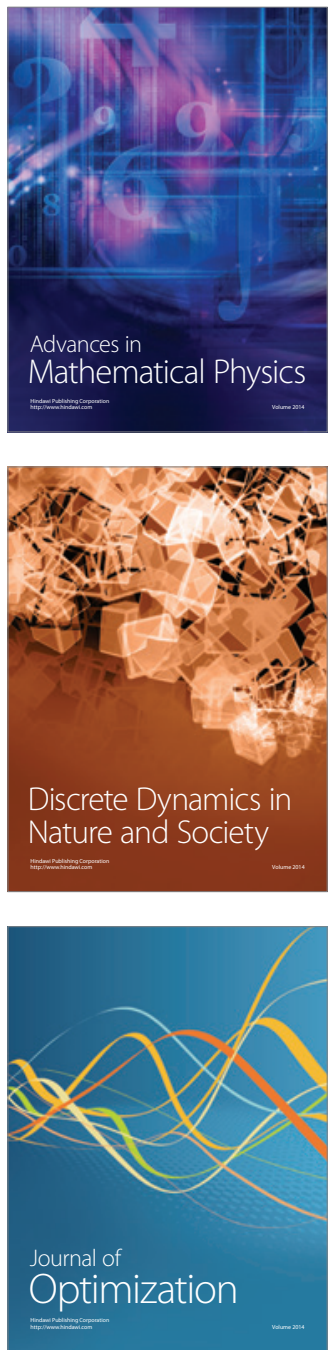